\title{
Knockdown of CDK6 enhances glioma sensitivity to chemotherapy
}

\author{
BING LI ${ }^{1,2^{*}}$, HUA HE$^{1 *}$, BANG-BAO TAO ${ }^{1 *}$, ZHEN-YU ZHAO ${ }^{3}$, GUO-HAN HU ${ }^{1}$, CHUN LUO $^{1}$, JU-XIANG CHEN ${ }^{1}$, \\ XUE-HUA DING ${ }^{1}$, PING SHENG ${ }^{1}$, YAN DONG ${ }^{1}$, LING ZHANG ${ }^{4}$ and YI-CHENG LU ${ }^{1}$ \\ ${ }^{1}$ Department of Neurosurgery, Changzheng Hospital, Second Affiliated Hospital of Second Military Medical \\ University, Shanghai 200003; ${ }^{2}$ Department of Neurosurgery, Wuxi Second People's Hospital, Wuxi, Jiangsu 214002; \\ ${ }^{3}$ Department of Neurosurgery, the 105th Hospital of PLA, Hefei, Anhui 230000; ${ }^{4}$ Editorial Department, \\ Chinese Journal of Neuromedicine, Zhujiang Hospital, Guangzhou, Guangdong 510282, P.R. China
}

Received February 13, 2012; Accepted April 3, 2012

DOI: $10.3892 /$ or.2012.1884

\begin{abstract}
Chemotherapy is widely used for the treatment of glioma. Given the high resistance of brain neoplasm tissues to chemotherapy, it is important to find new methods to improve the effects of chemotherapy. However, the molecular mechanisms underlying glioma resistance to chemotherapy are largely unknown. Here, we demonstrate that CDK6, a cell cycle regulator, is significantly upregulated in glioma cells, and the increasing expression of CDK6 correlates well with the grades of glioma malignancy. Using shRNA-mediated CDK6 knockdown, we found that the proliferation and survival of tumor cells were dramatically inhibited. Moreover, CDK6 knockdown in the U251 glioma cell line caused significant increase in the apoptosis of U251 cells treated with temozolomide (TMZ). Furthermore, CDK6 knockdown reduced the expression level of drug resistance genes such as MRP and MDR. These data indicate that CDK6 is an important mediator of glioma resistance to chemotherapy. Our findings provide a new strategy for the development of chemotherapy sensitizer.
\end{abstract}

\section{Introduction}

Glioma is the most common tumor of the brain and accounts for $45-55 \%$ of all brain tumors. Based on histological features (nuclear atypia, mitosis, microvascular enrichment and necrosis) and malignancy, glioma is classified into four grades (1). Grade 1 is innocent and curable with surgical resection; grade 2 is a low grade diffuse glioma and can progress into higher grades; grade 3 and 4 gliomas are malignant and have increased capability to proliferate and invade. The grade 4 glioma, glioblastoma multiforme (GBM), is the most aggressive.

Correspondence to: Dr Yi-Cheng Lu, Department of Neurosurgery, Changzheng Hospital, Second Affiliated Hospital of Second Military Medical University, 415 Fengyang Road, Shanghai 200003, P.R. China

E-mail: luyi_cheng@hotmail.com

*Contributed equally

Key words: CDK6, chemotherapy, glioma, drug resistance, MDR
Surgical resection is the primary therapy to treat glioma (2). However, surgical removal is very restrictive, thus it is difficult to completely remove diffusive tumor tissues, especially higher grade gliomas. These glioma tissues invade into the white matter or diffuse along the ventricle surface, so other strategies such as chemotherapy are very important to remove cancer cells as completely as possible (3-5). Chemotherapy increases the life of GBM patients. Clinical statistics reveals that, chemotherapy treatment leads to a $15 \%$ reduction of mortality, and a $6 \%$ increase of 1-more year survival. However, the effect of chemotherapy on glioma is not satisfactory, largely due to three problems. The first problem is the diagnosis of diffusive glioma tissues, which are tiny and grow deep in the brain tissue and are hard to access. The second problem is the blockade of chemotherapy drug by the blood-brain barrier. The third problem is that glioma is highly tolerant under chemotherapy treatment. Understanding the fundamentals, especially the molecular mechanisms, is critical to increase the susceptibility of glioma to chemotherapy.

Tumor cells originated from cells with abnormal proliferation and dysregulation of the cell cycle (6,7). Molecular profiling studies showed that the genes controlling cell growth and restricting cell division are silenced, and genes promoting cell proliferation and facilitating cell cycle are overexpressed. Many genes that promote cell cycle are found to be increased in transformed tumor cells (8). In cell cycle, CDK4 and 6 phosphorylate tumor suppressor Rb and promote $\mathrm{G} 1$ progression and G1/S transition (9). CDK6 is arrested and inhibited by INK4 and cyclin D (10). The CDK4/6 signaling was found to be highly activated in tumor cells, including sarcoma and leukemia $(11,12)$. Lam et al reported that 12 in 14 tumor samples showed increased expression of CDK6 (13). Moreover, mir-125b blocked G1/S transition and increased apoptosis by inhibiting CDK6 expression (14). Another microRNA mir-34a down-regulated CDK6 expression and significantly inhibited the proliferation of glioma cells (15). However, whether CDK6 mediates the chemotherapy resistance of glioma remains unknown.

In this study, we tested the hypothesis that increased expression and activation of CDK6 in glioma contribute to its resistance to chemotherapy treatment. Using immunohistochemistry, we found that the expression level of CDK6 correlated well with the tumor grade of gliomas. We then found that knocking down of CDK6 led to significant reduction on the cell cycle progression and proliferation of glioma cells. Finally, we found that glioma 
cells transfected with CDK6 shRNA showed increased apoptosis when exposed to temozolomide (TMZ) compared to cells transfected with control scRNA. Moreover, CDK6 knockdown resulted in down-regulated expression of several genes relating to drug resistance. Our findings suggested that CDK6 is an important molecular determinant contributing to chemotherapy resistance.

\section{Materials and methods}

Cell culture. The widely used glioma cell line U251 (ATCC, Manassas, VA) was cultured for our study. Cells were cultured in Dulbecco's modified Eagle's medium (DMEM) (Invitrogen, Carlsbad, CA), supplemented with $10 \%$ fetal bovine serum (Thermo Fisher Scientific, Inc., Waltham, MA) and antibiotics (penicillin and streptomycin, $50 \mathrm{U} / \mathrm{ml}$ each) (Invitrogen). Cells were grown in $60 \mathrm{~mm}$ dish and incubated in a humidified incubator with $5 \% \mathrm{CO}_{2}$ at $37^{\circ} \mathrm{C}$. Cells were inoculated at $1 \times 10^{4}$ cells/ dish, and were used at a confluent of $\sim 75 \%$. For chemotherapy treatment, cells were treated with TMZ (10 nM) for 24,48 or $72 \mathrm{~h}$.

Collection of human glioma and normal tissue samples. Surgically removed human glioma tissue samples and normal brain tissues were obtained frozen or paraffin-embedded from Changhai Hospital (China Secondary Military Medical University, Shanghai, China). Gliomas were graded by the Pathology Department of Changhai Hospital according to the World Health Organization grading system, presence and absence of nuclear atypia, mitosis, microvascular enrichment, and necrosis. Human normal brain tissues (mostly from the cortex) were obtained from patients with physical injuries to the brain. These specimens were collected from the patients registered at the above-mentioned hospitals, and written informed consent was obtained from the patients. The use of human tissues was approved by the ethics committees of the hospitals.

$R T-P C R$. Total RNA from mouse brain tissue or culture was extracted using TRIzol reagent (Invitrogen). The RNA was treated with DNase I to remove genomic DNA contamination and then reverse transcribed on a 96-well temperature cycler (ABI) using an M-MLV RT kit (Invitrogen). For PCR, a SYBR Premix Ex Taq kit (Takara) was used to prepare PCR solution and programs were run on Mastercycler Gradient PCR machine (Eppendorf) or Rotor-Gene 3000 Realtime Cycler (Corbett Research, Inc.). The primers used are: $c d k 6$ forward, GCGCC TATGGGAAGGTGTTC; $c d k 6$ reverse, TTGGGGTGCTC GAAGGTCT. $M d r-1$ forward, 5-TGGTTCAGGTGGCTCTG GAT-3, $m d r-1$ reverse,5-CTGTAGACAAACGATGAGCTATC ACA-3; $m r p$ forward, 5-GGCAAAGAAATAAAGCGACTG AA-3, $m r p$ reverse, 5-GGCTGTTGTCTCCATAGGCAAT-3.

Western blotting and immunohistology. For western blotting, total proteins were extracted from tissues and U251 culture using the RIPA buffer [10 mM Tris- $\mathrm{HCl}$ ( $\mathrm{pH} 7.4), 150 \mathrm{mM} \mathrm{NaCl}$, $1 \mathrm{mM}$ EDTA, $1 \%$ NP-40, $0.1 \%$ SDS, $0.5 \%$ sodium deoxycholate, $1 \mathrm{mM} \mathrm{Na} \mathrm{VO}_{4}, 1 \mathrm{mM}$ PMSF, and protease inhibitor cocktail]. Protein concentration was measured using BCA reagent (Pierce), diluted in sample loading buffer and subjected to SDS-PAGE gel electrophoresis, then transferred to PVDF membrane $(0.45 \mu \mathrm{m}$,
PALL), and blocked by $0.05 \%$ TBST with $5 \%$ milk and then blotted with primary antibodies at $4^{\circ} \mathrm{C}$ overnight and secondary antibodies at room temperature for $1 \mathrm{~h}$. Bound antibodies were detected by the ECL immunoblotting detection reagent (GE Healthcare) and exposed to X-ray films. Band densities were quantified by Gel-Pro Analyzer, and the densitometric results are shown. The relative amount of proteins was determined by normalizing the density value of target protein to internal control and the external control.

For immunohistochemistry, $4 \mu \mathrm{m}$ thickness paraffinprocessed sections were mounted on poly-D-lysine-coated glass slides. Each slide was dewaxed in $100 \%$ xylene and rehydrated by incubation in decreasing concentrations $(100,95$ and $75 \%)$ of alcohol. Sections were incubated in $3 \%$ hydrogen peroxide to quench endogenous peroxidase. Antigen retrieval was performed in a microwave oven $\left(95^{\circ} \mathrm{C}\right.$ for $\left.15 \mathrm{~min}\right)$. The immunoreactions were performed with $\mathrm{ABC}$ kit (Maixin Biotech, China). Briefly, sections were blocked with horse serum and incubated overnight with diluted mouse anti-human CDK6 antibody (Santa Cruz) at $4^{\circ} \mathrm{C}$. Following washes with TBST $(3 \times 5 \mathrm{~min})$, the slides were incubated with the appropriate anti-mouse IgG (Dako Envision) at room temperature for $30 \mathrm{~min}$, and then washed with TBST (3x5 min). Sections were developed using DAB reagent and counterstained with hematoxylin to stain the nucleus. Sections were dehydrated by incubating in increasing concentrations (75, 95 and $100 \%$ ) of alcohol and in $100 \%$ xylene before coverslips were mounted onto the sections.

RNAi and transfection. To knockdown $c d k 6$, three siRNA targeting sequences were designed using online siRNA Target Finder (Ambion). The three sets of siRNA was synthesized and annealed to form double strand siRNA and transfected into U251 cells by Lipofectamine 2000 (Invitrogen). The knockdown efficiency was validated by RT-PCR and Western blotting, and finally the most specific and efficient targeting sequence was used for functional assay. The sequence is: 5'-CATGTCGATCAAGACTTGA-3'. For vector based shRNA construct, an shRNA with the selected targeting sequence was cloned into pSuper-GFP or pLVTHM. For transfection of $\mathrm{U} 251$ cells, $4 \mu \mathrm{g}$ of shRNA or control plasmid was dissolved in Opti-MEM and coated with Lipofectamine 2000 reagent for a 60-mm dish. Six hours later, the culture medium was replaced, and $48 \mathrm{~h}$ later, the cells were subjected for analysis.

MTT assay. Cells were cultured in a 96-well plate at a density of $5 \times 10^{3}$. Two hours before examination, cell-counting kit- 8 reagent (10 $\mu \mathrm{l} /$ well) was added into the wells and incubated for $2 \mathrm{~h}$. The reaction was terminated by adding SDS solution $(0.1 \%$ final concentration). Before reading, the plate was shaked by the supplied plate shaker and then read at $450 \mathrm{~nm}$.

Flow cytometry to examine cell cycle progression. Flow cytometry assays were used to study the effect of knocking down CDK6 cell cycle progression. U251 cells were harvested, treated with $0.25 \%$ trypsin, washed in PBS, centrifuged (1500 rpm, $5 \mathrm{~min}$ ) and resuspended with binding buffer. The cell density was adjusted to $3 \times 10^{5}$ cells $/ \mathrm{ml}$. Cell suspension $(195 \mu \mathrm{l})$ and $5 \mu$ l Annexin V/FITC were combined and incubated at RT for $10 \mathrm{~min}$. Next, propidium iodide was added to a final concentration of $1 \mu \mathrm{g} / \mathrm{ml}$ and incubated at $37^{\circ} \mathrm{C}$ for $30 \mathrm{~min}$. In each sample, 

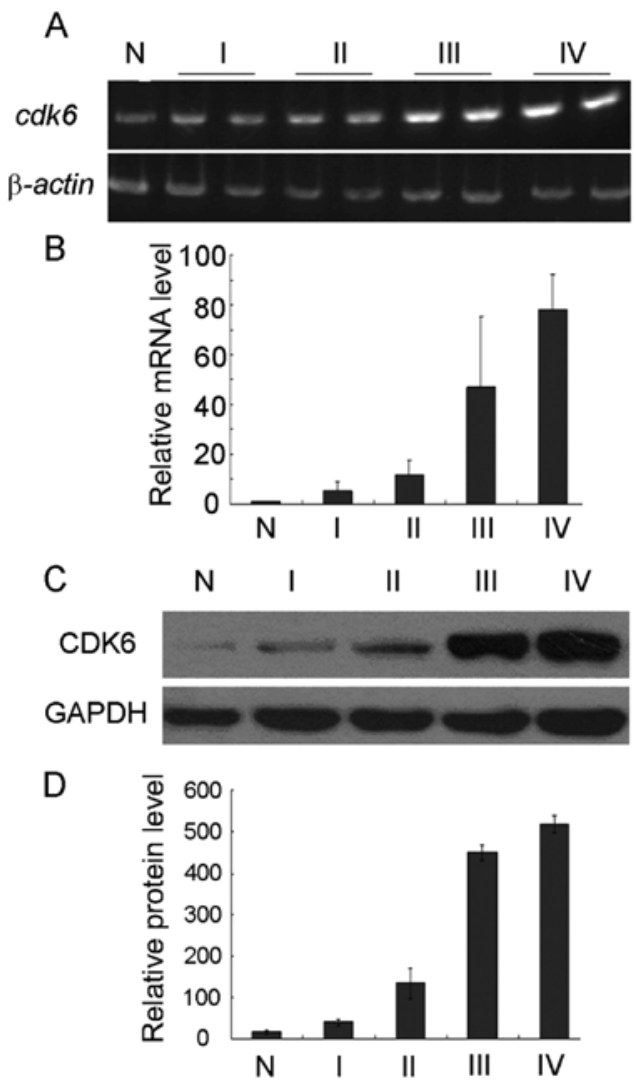

Figure 1. CDK6 expression is upregulated in different grades of gliomas. (A and B) Upregulation of $c d k 6$ mRNA in glioma cells. $\mathrm{N}$, normal brain tissue; I, grade I glioma tissue, classified according to WHO standards; II, WHO grade II glioma tissue; III, WHO grade III glioma tissue; IV, WHO grade IV glioma tissue. (C and D) Upregulation of CDK6 protein in glioma cells.

$6 \times 10^{4}$ cells were assayed on FACSCalibur (Becton-Dickinson, Franklin Lakes, NJ) and the cell cycle phase distribution was analyzed by CellQuest Pro software (Becton-Dickinson).

Statistical analysis. The data are presented as mean \pm SD. All statistical analysis were done using One-way ANOVA and SPSS software.

\section{Results}

Significant upregulation of CDK6 in glioma. Although CDK6 expression was reported to be increased in gliomas, it remains unclear whether the malignant grade correlates with the upregulation of CDK6 expression. To examine this, we collected 34 glioma samples at different grades and 4 samples of normal brain tissue. The mRNA of CDK6 showed good correlation with different grades of gliomas (normal tissue, 1.02 \pm 0.25 ; grade 1, 5.50 \pm 0.25 ; grade 2, 11.78 \pm 5.90 ; grade 3, 46.74 \pm 28.81 ; grade 4, 78.11 \pm 14.04 , Fig. 1A and B). The expression change of CDK6 protein was also studied using Western blotting, and similar upregulation pattern was found in glioma samples (Fig. 1C and D). In normal tissue, the protein level of CDK6 was 16.81 \pm 3.25 , whereas in grade 1 glioma, CDK6 expression was increased to $41.33 \pm 7.38$, and in grade 2 glioma to $35.11 \pm 37.54$. In highly malignant glioma, significant elevation of CDK6 protein was observed (grade 3, 449.45 \pm 20.17 ; grade 4, 518.61 \pm 19.61 ). Furthermore, immunostaining showed that, CDK6 was kept at much low level in normal brain tissue (Fig. 2A). In grade 1 glioma tissue, CDK6 was increased moderately (Fig. 2B). In grade 2 glioma, CDK6 was highly upregulated (Fig. 2C), whereas in more malignant grade 3 or 4 glioma, higher upregulation of CDK6 expression was observed (Fig. 2D).

We also employed microarray chips to do whole-genome comparison of gene expression profiles between glioma and normal tissues. Among those highly upregulated genes in glioma tissue, CDK6 also showed good correlation with glioma grades and survival time according to follow-up visit data of patients (data not shown).

Knockdown of CDK6 mRNA in glioma inhibits glioma growth. Increased high level of CDK6 may promote the G1 progression and $\mathrm{G} 1 / \mathrm{S}$ transition, thus leads to uncontrolled cell division and proliferation, and finally tumorigenesis and malignancy $(13,16,17)$. Although upregulation of CDK6 expression correlated with glioma grades, whether CDK6 contributed to the higher proliferation and invasion capability requires further determination.

We used U251 cell line to test whether CDK6 played a role in regulating glioma proliferation (Fig. 3A). To knock down CDK6, an shRNA was cloned into pSuper-GFP construct, and transfected into U251 cells (Fig. 3B), 72 h later, cell cycle distribution of U251 cells was analyzed by flow cytometry. Compared to scrambled shRNA (scRNA), $c d k 6$-targeting shRNA (shRNA) resulted in significantly increased retention of $\mathrm{U} 251$ cells in G1 phase (scRNA, 51.07 $\pm 1.33 ; c d k 6$ shRNA, 59.77 $\pm 1.85 ; \mathrm{p}<0.05$, Fig. 3D and E), thereby leading to a great reduction of the ratio of cells in G2/M phase (scRNA, 25.93 \pm 0.50 ; $c d k 6$ shRNA, 21.85 $\pm 1.32 ; \mathrm{p}<0.05$, Fig. 3D and E).

Knocking down $c d k 6$ inhibited cell cycle progression, thus blocking glioma proliferation and tumor growth. To examine the function of $c d k 6$ in cell growth, we used MTT assay to analyze U251 cells after shRNA transfection (Fig. 3C). Compared to control scRNA, $c d k 6$ shRNA expression greatly reduced the MTT absorption value (scRNA, 0.613 \pm 0.075 ; $c d k 6$ shRNA, $0.417 \pm 0.065 ; \mathrm{p}<0.05)$, indicating significant inhibition of U251 cell growth by RNAi of CDK6.

Enhanced chemotherapy effect of TMZ on CDK6-knockdown glioma. Chemotherapy is an indispensible treatment for higher grade gliomas $(4,18)$. However, the low permeability of drugs across blood-brain barrier reduced drug concentration in cerebral-spinal fluid. On the other hand, glioma cell are resistant to chemotherapy treatment, mainly due to high activity of DNA repair mechanism, tolerance to cell cycle check points, reduced uptake of drug and quick metabolism of drug molecules (18-20). In the above studies, we found that CDK6 expression is highly upregulated in glioma cells. As CDK6 is a transducer of cytoplasm signal to nuclear transcription activity and DNA repair mechanism, we speculated that the chemotherapy-resistance of glioma is mainly mediated by increased level of CDK6.

To test this hypothesis, we first established a culture model of chemotherapy. Temozolomide (TMZ) is an alkylating agent and effective for the treatment of higher grade glioma (5). We thus tested the effect of TMZ on glioma in vitro (Fig. 4). Compared to control reagent, TMZ showed inhibition of U251 cell growth even at $24 \mathrm{~h}$ after treatment (con, $0.111 \pm 0.012$; TMZ, $0.098 \pm 0.010$ ). At $48 \mathrm{~h}$, significant inhibition of $\mathrm{U} 251$ cell growth 

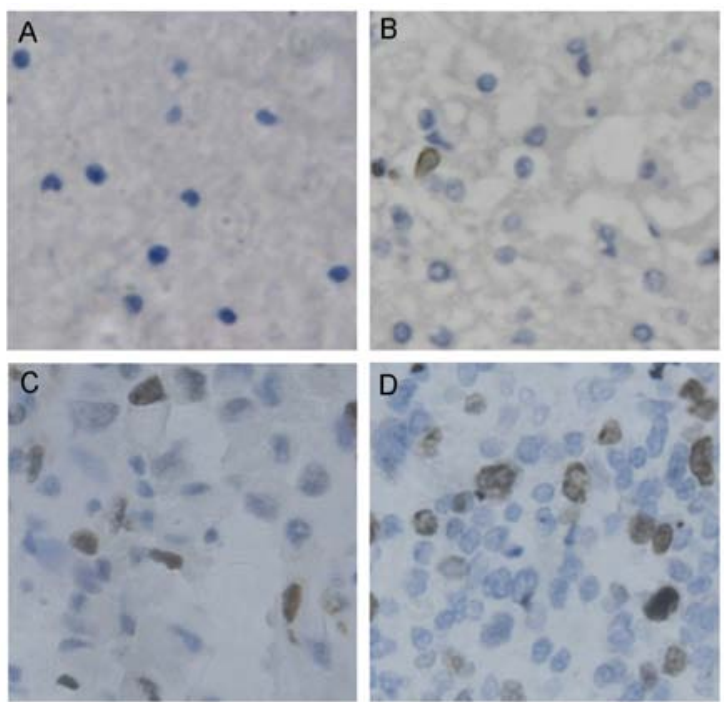

Figure 2. CDK6 immunostaining density is increased in different grades of glioma tissues. (A) No staining for CDK6 was noted in PA (WHO-I); (B) Few CDK6-positive tumor cells in a diffuse astrocytoma (WHO-II); (C) Higher immunostaining intensity was detected in anaplastic astrocytoma (WHO-III); (D) Strong immunostaining was detected in glioblastoma (WHO-IV). by TMZ can be observed (con, $0.286 \pm 0.036$; TMZ, $0.186 \pm 0.006$; $\mathrm{P}<0.01)$. Next, we compared the effect of combined treatment of $\mathrm{U} 251$ cells with TMZ and $c d k 6$ knockdown to single treatment with TMZ. Interestingly, combined treatment of U251 cells with TMZ and $c d k 6$ shRNA resulted in significant decrease of U251 cell growth at $48 \mathrm{~h}$ after treatment $(0.155 \pm 0.018, \mathrm{p}<0.05)$, lower than single treatment with either TMZ $(0.186 \pm 0.006)$ or $c d k 6$ shRNA $(0.177 \pm 0.017)$. These results strongly suggested that increased expression of CDK6 contributed to the resistance of glioma to chemotherapy.

Reduced drug-resistance to chemotherapy in CDK6-knockdown glioma. The facilitation of TMZ-mediated chemotherapy toxicity on U251 glioma cell growth by CDK6 knockdown indicated that aberrant cell signaling of glioma cells underlies the resistance of glioma to chemotherapy. To test whether CDK6 knockdown had an effect on drug resistance-related genes, we analyzed the expression change of several key genes by RT-PCR and western blotting. MDR-1 is a transmembrane glycoprotein of the ATP-binding cassette superfamily (21). MDR-1 acts as a bump to transport drug molecules out of the cells, thereby reducing

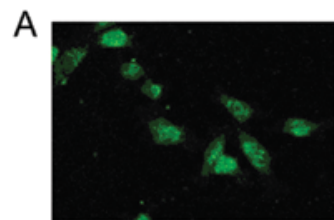

B
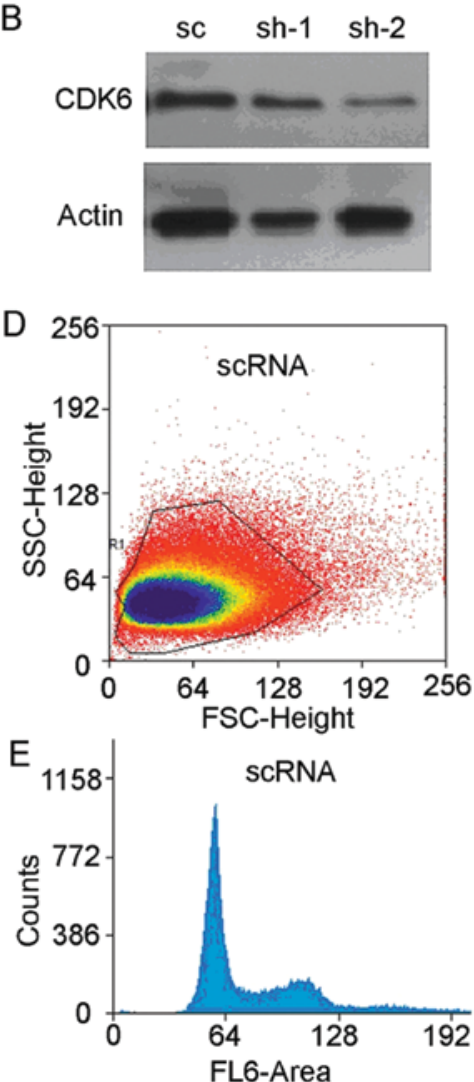
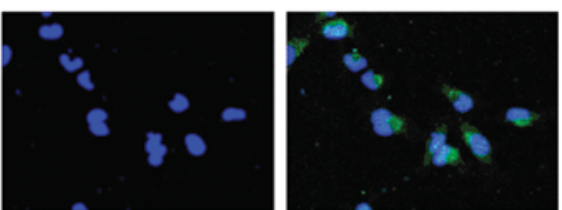

C
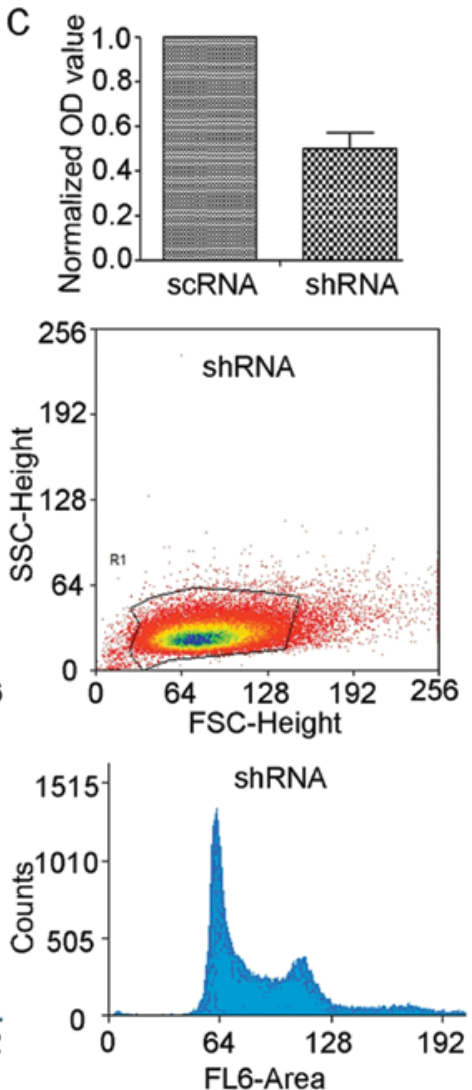

Figure 3. CDK6 knockdown leads to cell cycle retention and reduced glioma growth. (A) CDK6 immunofluorescence in U251 glioma cells. Blue, DAPI; (B) Examination of CDK6 RNAi shRNA by western blotting. (C) CDK6 knockdown reduced cell growth ability of U251 cells measured by MTT assay (450 nm). (D and E) CDK6 knockdown led to cell cycle retention and G1 phase measured by flow cytometry. Data are shown in dot plot format (D) or histogram format (E). Left, scrambled control shRNA(scRNA); right, $c d k 6$ shRNA. 
A

\begin{tabular}{lcccc} 
A & \multicolumn{5}{c}{ MTT assay Absorbance value $(450 \mathrm{~nm})$} \\
\hline sham & $0 \mathrm{~h}$ & $24 \mathrm{~h}$ & $48 \mathrm{~h}$ & $72 \mathrm{~h}$ \\
\hline Veh+scRNA & $0.50 \pm 0.007$ & $0.111 \pm 0.012$ & $0.286 \pm 0.036$ & $0.622 \pm 0.027$ \\
\hline Veh+shRNA & $0.047 \pm 0.003$ & $0.109 \pm 0.012$ & $0.326 \pm 0.048$ & $0.613 \pm 0.019$ \\
\hline TMZ+scRNA & $0.051 \pm 0.004$ & $0.091 \pm 0.009$ & $0.186 \pm 0.006$ & $0.289 \pm 0.035$ \\
\hline TMZ+shRNA & $0.051 \pm 0.007$ & $0.081 \pm 0.007$ & $0.155 \pm 0.018$ & $0.298 \pm 0.016$ \\
\hline
\end{tabular}

B

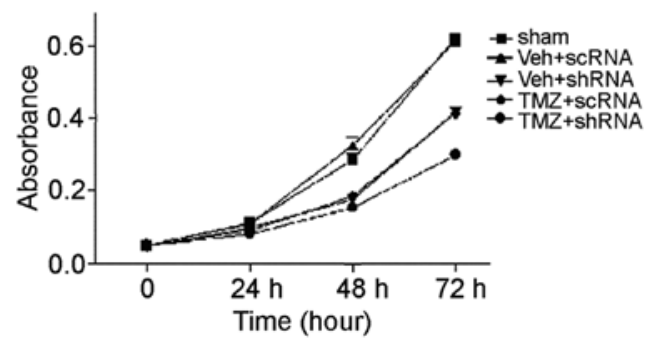

Figure 4. CDK6 knockdown enhances TMZ-mediated inhibition of glioma cell survival and growth. (A) Relative cell survival and growth value revealed by OD value derived from MTT assay. (B) Statistical table showing glioma cell number of U251 cells with different treatments. Veh, vehicle that dissolve TMZ (temozolomide).

drug toxicity to cancer cells. In $c d k 6$-knockdown cells, MDR-1 expression was greatly down-regulated (shRNA, 0.44 \pm 0.18 ; scRNA, 3.07 \pm 0.49 ; sham, 3.09 \pm 0.27 ; p $<0.01$, Fig. 5A). MRP is another member of the ABC transporter superfamily (22). We also found that MRP expression in $c d k 6$-knockdown U251 cells was lower than scRNA transfected cells (shRNA, 1.93 \pm 1.40 ; scRNA, 7.27 \pm 1.49 ; sham, 8.39 \pm 1.17 ; p <0.01, Fig. 5B).

Drug resistance can also result from high level of DNA repair activity. PARP is a DNA polymerase which plays an important role in DNA repair (23). PARP can detect DNA single-strand break and bind to the break sequence and initiate the repair process by the synthesis of a poly(ADP-ribose) chain and recruiting other DNA repair proteins. PARP1 is highly expressed in glioma (24), and its expression can be increased by TMZ treatment. In turn, the upregulated PARP1 protects glioma cells from DNA-damage induced cell cycle retention and apoptosis. In $c d k 6$-knockdown cells, PARP1 protein was significantly down-regulated (scRNA, 623.77 \pm 15.36 ; $c d k 6$ shRNA, 237.23 \pm 28.09 ; p <0.05, Fig. 5C and D). Conclusively, increased level of CDK6 resulted in enhanced expression of several key genes responsible for drug resistance, and CDK6 knockdown or inhibition was effective in reducing their expression, thereby reducing chemotherapy drug-resistance of glioma cells.

\section{Discussion}

Gene mutation and their aberrant expression are very common in tumor cells. Microarray studies revealed that in each type of tumor cells, more than one hundred genes exhibited abnormal expression or mutation (25). Thus, finding the key genes that participate in or regulate the tumorigenesis process has become an important task in cancer research. CDK6 regulates cell cycle progression, especially in the transition from G1 to $S$ phase (9). We first demonstrated that CDK6 upregulation correlated with the malignant grades of glioma, which implied that the expression level of CDK6 might determine the proliferation and invasion capability. As an upstream regulator of cancer repressor $\mathrm{Rb}, \mathrm{CDK} 6$ expression level is an indicator of cell division activity (16). CDK6 phosphorylates $\mathrm{Rb}$ and activates transcription factor E2F1/2, thus pushing cells into $\mathrm{S}$ phase and triggering DNA synthesis (6).

The control of G1/S transition is the first gate to cell division, thus becomes extremely important for cell cycle control. The highly upregulated CDK6 in glioma cells make the G1/S checkpoint permissive, thereby enabling cell proliferation. We found that $c d k 6$ knockdown resulted in significant decrease of cell growth, indicating that CDK6 is an organizer of cell cycle, and higher expression of CDK6 may underlie the chemotherapy resistance of glioma. As a kinase, CDK6 activity can easily be inhibited by small molecules, which can pass across the blood-
A

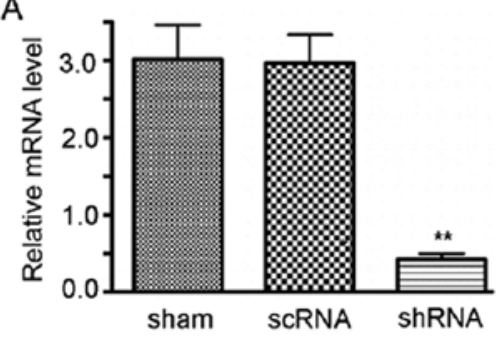

B

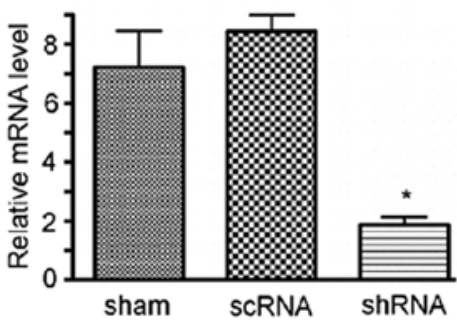

C

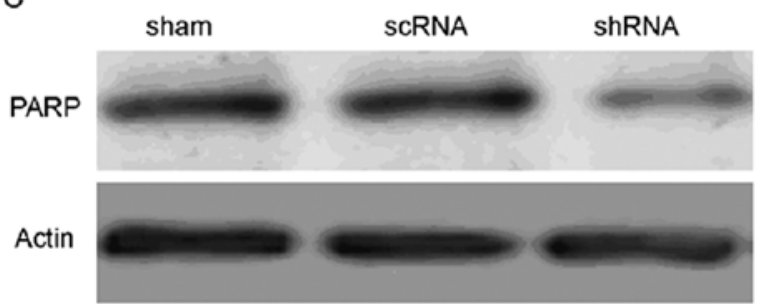

D

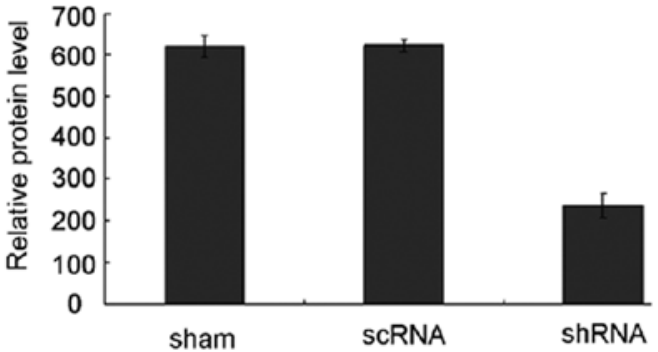

Figure 5. Expression of drug-resistant genes is significantly down-regulated in CDK6-knockdown glioma cells. (A) Decreased expression of MDR1 in CDK6 knockdown U251 cells. Sham, empty vector without shRNA; scRNA, scrambled shRNA; shRNA, $c d k 6$ shRNA. (B) Down-regulated expression of MRP in CDK6 knockdown U251 cells. (C) Protein level of PARP1 was significantly reduced in CDK6 knockdown U251 cells examined by western blotting. (D) Statistical analysis of western blotting result of PARP1. ${ }^{*} \mathrm{p}<0.05 ;{ }^{* *} \mathrm{p}<0.01$. 
brain barrier. Many drug-resistant genes are upregulated in glioma cells, some of which may be downstream genes of E2F transcription activator complex $(26,27)$. Therefore, inhibiting CDK6 may have considerable effect on enhancing the sensitivity of glioma cells to chemotherapy drugs. In our study, we observed that combined treatment of glioma cells with chemotherapy drug TMZ and CDK6 shRNA significantly inhibited cell growth, which is more effective than single treatment either with TMZ or CDK6 shRNA.

The mechanism underlying chemotherapy-resistance of glioma has been intensively studied $(18,19,21,22)$. Many drugresistant genes have been found, which mainly belongs to DNA repair enzymes, small-molecule transmembrane transporters and drug metabolism proteins. Given their wide divergence and differential protein structures, it may not be practicable to interfere with a single drug-resistant gene. Therefore, finding the upstream regulators maybe more effective to increase drug uptake and toxicity. CDK6 knockdown resulted in significant down-regulation of MDR1, MRP and PARP, indicating that CDK6 is an upstream activator of these drug-resistance related genes, and should be a promising target to reduce drug-resistance of glioma cells. Future work will be needed to study how CDK6 overexpression leads to the upregulation of these genes.

\section{Acknowledgements}

This study was supported by grants from the Key Foundation Project of the National Natural Science Foundation of China (no. 30930094).

\section{References}

1. Behin A, Hoang-Xuan K, Carpentier AF and Delattre J-Y: Primary brain tumours in adults. Lancet 361: 323-331, 2003.

2. Lacroix M, Abi-Said D, Fourney DR, et al: A multivariate analysis of 416 patients with glioblastoma multiforme: prognosis, extent of resection, and survival. J Neurosurg 95: 190-198, 2001.

3. Stewart L: Chemotherapy in adult high-grade glioma: a systematic review and meta-analysis of individual patient data from 12 randomised trials. Lancet 359: 1011-1018, 2002.

4. Reardon DA, Rich JN, Friedman HS and Bigner DD: Recent advances in the treatment of malignant astrocytoma. J Clin Oncol 24: 1253-1265, 2006.

5. Stupp R, Dietrich P-Y, Kraljevic SO, et al: Promising survival for patients with newly diagnosed glioblastoma multiforme treated with concomitant radiation plus temozolomide followed by adjuvant temozolomide. J Clin Oncol 20: 1375-1382, 2002.

6. Malumbres M and Barbacid M: Cell cycle, CDKs and cancer: a changing paradigm. Nat Rev Cancer 9: 153-166, 2009.

7. Schwartz GK and Shah MA: Targeting the cell cycle: a new approach to cancer therapy. J Clin Oncol 23: 9408-9421, 2005.

8. Deshpande A, Sicinski P and Hinds PW: Cyclins and cdks in development and cancer: a perspective. Oncogene 24: 2909-2915, 2005.
9. Harbour JW, Luo RX, Santi AD, Postigo AA and Dean DC: Cdk phosphorylation triggers sequential intramolecular interactions that progressively block Rb functions as cells move through G1 . Cell 98: 859-869, 1999.

10. Ezhevsky SA, Nagahara H, Vocero-Akbani AM, Gius DR, Wei MC and Dowdy SF: Hypo-phosphorylation of the retinoblastoma protein (pRb) by cyclin D:Cdk4/6 complexes results in active pRb. Proc Natl Acad Sci USA 94: 10699-10704, 1997.

11. Hayette S, Tigaud I, Callet-Bauchu E, et al: In B-cell chronic lymphocytic leukemias, 7q21 translocations lead to overexpression of the CDK6 gene. Blood 102: 1549-1550, 2003.

12. Van Dross R, Yao S, Asad S, et al: Constitutively active K-cyclin/ cdk6 kinase in Kaposi sarcoma-associated herpesvirus-infected cells. J Natl Cancer Inst 97: 656-666, 2005.

13. Lam PY, Di Tomaso E, Ng HK, Pang JC, Roussel MF and Hjelm NM: Expression of p19INK4d, CDK4, CDK6 in glioblastoma multiforme. Br J Neurosurg 14: 28-32, 2000.

14. Shi L, Zhang J, Pan T, et al: MiR-125b is critical for the suppression of human U251 glioma stem cell proliferation. Brain Res 1312: $120-126,2010$.

15. Li Y, Guessous F, Zhang Y, et al: MicroRNA-34a inhibits glioblastoma growth by targeting multiple oncogenes. Cancer Res 69: 7569-7576, 2009.

16. Mendrzyk F, Radlwimmer B, Joos S, et al: Genomic and protein expression profiling identifies CDK6 as novel independent prognostic marker in medulloblastoma. J Clin Oncol 23: 8853-8862, 2005.

17. Easton J, Wei T, Lahti JM and Kidd VJ: Disruption of the cyclin D/ cyclin-dependent kinase/INK4/retinoblastoma protein regulatory pathway in human neuroblastoma. Cancer Res 58: 2624-2632, 1998.

18. Bredel $\mathrm{M}$ and Zentner J: Brain-tumour drug resistance: the bare essentials. Lancet Oncol 3: 397-406, 2002.

19. Feun LG, Savaraj N and Landy HJ: Drug resistance in brain tumors. J Neurooncol 20: 165-176, 1994.

20. Bredel M: Anticancer drug resistance in primary human brain tumors. Brain Res Rev 35: 161-204, 2001.

21. Declèves X, Fajac A, Lehmann-Che J, et al: Molecular and functional MDR1-Pgp and MRPs expression in human glioblastoma multiforme cell lines. Int J Cancer 98: 173-180, 2002.

22. Haga S, Hinoshita E, Ikezaki K, et al: Involvement of the multidrug resistance protein 3 in drug sensitivity and its expression inhuman glioma. Cancer Sci 92: 211-219, 2001.

23. de Murcia JM, Niedergang C, Trucco C, et al: Requirement of poly(ADP-ribose) polymerase in recovery from DNA damage in mice and in cells. Proc Natl Acad Sci USA 94: 7303-7307, 1997.

24. Cheng CL, Johnson SP, Keir ST, et al: Poly(ADP-ribose) polymerase-1 inhibition reverses temozolomide resistance in a DNA mismatch repair-deficient malignant glioma xenograft. Mol Cancer Ther 4: 1364-1368, 2005.

25. Rhee CH, Hess K, Jabbur J, Ruiz M, Yang Y, Chen S, Chenchik A, Fuller GN and Zhang W: cDNA expression array reveals heterogeneous gene expression profiles in three glioblastoma cell lines. Oncogene 18: 2711-2717, 1999.

26. Johnson DG, Schwarz JK, Cress WD and Nevins JR: Expression of transcription factor E2F1 induces quiescent cells to enter $\mathrm{S}$ phase. Nature 365: 349-352, 1993.

27. Chen H-Z, Tsai S-Y and Leone G: Emerging roles of E2Fs in cancer: an exit from cell cycle control. Nat Rev Cancer 9: 785-797, 2009. 